\title{
A influência da hiperglicemia como fator prognóstico em pacientes com hematoma intraparenquimatoso encefálico espontâneo relacionado à hipertensão arterial
}

Lucas Perez de Vasconcellos ${ }^{1}$, José Carlos Esteves Veiga ${ }^{2}$, Juan Antonio Castro Flores ${ }^{3}$, Bechara Mattar Neto ${ }^{3}$

Disciplina de Neurocirurgia do Departamento de Cirurgia da Faculdade de Ciências Médicas da Santa Casa de São Paulo, SP, Brasil

\section{RESUMO}

Objetivo: Examinar a relação da hiperglicemia e da pressão arterial com o prognóstico neurológico de pacientes com hematoma intraparenquimatoso encefálico espontâneo, relacionado à hipertensão arterial. Método: Estudamos, retrospectivamente, a evolução clínica de 100 pacientes com hematoma intraparenquimatoso encefálico espontâneo submetidos ao tratamento cirúrgico para drenagem do hematoma, na Santa Casa de São Paulo, no período de janeiro de 2000 a dezembro de 2004. Os pacientes foram subdivididos em dois grupos: um grupo de evolução favorável (escores 4 e 5 na Escala de Prognóstico Neurológico) e outro desfavorável (escores 1, 2 e 3 na Escala de Prognóstico Neurológico). Resultados: Os doentes com evolução neurológica desfavorável apresentaram níveis significativamente maiores de glicemia e de pressão arterial, tanto na admissão quanto na evolução pós-operatória. Os pacientes com prognóstico neurológico favorável foram significativamente mais jovens, admitidos com a pontuação na Escala de Coma de Glasgow significativamente maior (em média, acima de 12) e apresentaram período de internação mais prolongado. Conclusão: A hiperglicemia e a hipertensão arterial sistêmica são componentes freqüentes nas lesões neurológicas, sendo indicadores significativos de gravidade e confiáveis para a avaliação do prognóstico neurológico.

\section{PALAVRAS-CHAVE}

Hematoma intraparenquimatoso encefálico espontâneo. Hiperglicemia. Prognóstico neurológico.

\section{ABSTRACT}

The influence of hyperglycemia on neurological outcome in patients with primary spontaneous intracerebral hematoma related to hypertension

Objective: To study the relationship of hyperglycemia and hypertension with neurological outcome after spontaneous intracerebral hematoma. Method: We retrospectively studied 100 patients with primary spontaneous intracerebral hematoma related to arterial hypertension treated surgically for evacuation of the hematoma in the Neurosurgical Department of Santa Casa de São Paulo, between January 2000 and December 2004. The patients were separated in two groups based on their Glasgow Outcome Scale score. The favorable category included patients with Glasgow Outcome Scale scores 5 or 4, and the unfavorable outcome category included those with scores 1, 2 and 3. Results: Patients with unfavorable outcome had significantly higher levels of serum glucose and blood pressure, both at the admission and in the postoperative. The patients with favorable category were significantly younger, admitted with a Glasgow Coma Scale score significantly higher and had a longer hospitalization period. Conclusion: The hyperglycemia and hypertension, frequent components of the stress response to brain damage, are significant indicators of its severity, and reliable predictors of neurological outcome.

\section{KEY WORDS}

Primary intracerebral hematoma. Hyperglicemia. Neurological outcome.

1 Residente de segundo ano do Serviço de Neurocirurgia dos Hospitais da Santa Casa de Misericórdia de São Paulo, SP.

2 Professor adjunto e chefe do Serviço de Neurocirurgia dos Hospitais da Santa Casa de Misericórdia de São Paulo.

3 Médico assistente do Serviço de Neurocirurgia dos Hospitais da Santa Casa de Misericórdia de São Paulo. 


\section{Introdução}

Os hematomas intraparenquimatosos encefálicos espontâneos (HIPE) representam 10\% a 20\% das doenças encefalovasculares nos Estados Unidos ${ }^{13,24}$. Embora sua proporção em relação aos acidentes vasculares cerebrais possa parecer baixa, os HIPE apresentam elevadas taxas de morbidade e mortalidade. Nos primeiros trinta dias desde a instalação, o índice de mortalidade é de 35\% a $50 \%$, duas a seis vezes maior que o observado em acidentes vasculares cerebrais isquêmicos (AVCI), com mais da metade dos óbitos ocorrendo nos dois primeiros dias e em $6 \%$ dos pacientes antes de chegar aos hospitais ${ }^{7-9,21,26}$.

Dentre os fatores de risco associados aos HIPE, destacam-se: idade avançada, hipertensão arterial (HA), hiperglicemia, angiopatia amilóide, uso de drogas, malformações vasculares arteriovenosas (MAV), angiomas cavernosos, iatrogenias (uso de trombolíticos ou anticoagulantes), neoplasias encefálicas, transformações hemorrágicas de AVCI e discrasias sanguíneas?.

A localização do hematoma é de extrema importância, pois pode predizer o prognóstico, assim como influenciar no tipo de tratamento ${ }^{1,3}$. Os HIPE podem ser classificados em: profundos, situados mais comumente nos gânglios da base; superficiais ou lobares, situados na transição cortico-subcortical; infratentoriais, quando situados no tronco encefálico ou no cerebelo.

Apesar de todo o conhecimento adquirido em relação à fisiopatologia e à etiopatogenia dos HIPE, o tratamento ideal ainda permanece cercado de muitas controvérsias. Quando indicado, o tratamento cirúrgico inclui drenagem por craniotomia, ou por punção aspirativa, ou por sondagem via endoscópica.

O presente estudo tem como objetivo analisar de maneira retrospectiva 100 casos de HIPE tratados cirurgicamente, procurando determinar uma relação entre níveis pressóricos e glicêmicos, aspectos clínicos e epidemiológicos, com a evolução e o prognóstico neurológico.

\section{Casuística e método}

Foram analisados de forma retrospectiva 100 prontuários médicos consecutivos de pacientes com hematomas intraparenquimatosos espontâneos relacionados à HA, submetidos ao tratamento cirúrgico pelo Serviço de Neurocirurgia da Santa Casa de São Paulo entre janeiro de 2000 e fevereiro de 2005. Os limites de variação de idade foram de 8 a 89 anos (média de 50,24 anos), sendo $66 \%$ dos pacientes do sexo masculino. Quanto à etnia, 68 pacientes eram brancos, 30 negros e 2 amarelos.
A presença de trauma craniencefálico (TCE), hematoma, ruptura de aneurisma, politraumatismo, história pregressa de algum procedimento neurocirúrgico e óbito em até 24 horas após a admissão foram critérios de exclusão desta casuística.

Todos os pacientes foram examinados no Serviço de Emergência por um neurocirurgião. À admissão, os seguintes valores foram averiguados e anotados: escore na Escala de Coma de Glasgow (ECGl) ${ }^{10}$, pressão arterial média (PAM $=2 \times$ PA diastólica + PA sistólica $\div 3$ ) e glicemia.

Após o tratamento inicial e a estabilização clínica, todos os pacientes realizaram tomografia computadorizada craniencefálica (TC), foram investigados quanto à causa e submetidos a tratamento cirúrgico. O diagnóstico etiológico da hemorragia foi investigado por exames pré-operatórios (arteriografia), durante o ato operatório por visualização direta, ou no pós-operatório, quando havia suspeita de MAV, por exame anatomopatológico dos coágulos removidos.

Decorridas 24 horas após a cirurgia, foram analisados os valores da PAM, da glicemia e da pontuação na ECGl.

No momento da alta hospitalar, os pacientes foram divididos em dois grupos com base na Escala de Prognóstico de Glasgow (GOS) ${ }^{17}$. Um grupo incluiu pacientes com evolução favorável, com recuperação completa ou mantendo déficits moderados (GOS $=5$ e 4 respectivamente), enquanto o outro incluiu aqueles que tiveram evolução desfavorável, com incapacidade grave, em estado vegetativo persistente ou óbito ( $\mathrm{GOS}=3,2$ e 1 respectivamente).

Os valores das variáveis estudadas foram apresentados juntamente com sua média e desvio-padrão.

O estudo estatístico dos resultados obtidos nos dois grupos foi realizado pelo teste T de Student, controlado pelo teste de Levene para igualdade de variâncias, utilizando o programa SPSS (Statistical Package for Social Sciences) em sua versão 10.0. Foram considerados significativos os valores de $\mathrm{p}<$ 0,05 para testes bicaudais.

\section{Resultados}

A idade e a distribuição segundo gênero, ECGl, níveis glicêmicos, PAM, tempo de internação, localização do hematoma e prognóstico neurológico estão listados na tabela 1. A análise linear de correlação pode determinar as diferenças estatísticas entre os dois grupos com relação a glicemia, PAM e ECGl de entrada, bem como a valores obtidos 24 horas após a cirurgia, idade dos pacientes e número total de dias internados. 
Tabela 1.

Dados demográficos, tempo de internação,

ECGl, glicemia, PAM, localização e prognóstico neurológico dos 100 pacientes do estudo*

\begin{tabular}{|c|c|c|}
\hline & GOS $\leq 3$ & GOS $\geq 4$ \\
\hline № de casos & 60 & 40 \\
\hline Gênero (M/F) & $39 / 21$ & $27 / 13$ \\
\hline Idade (média \pm DP) $)^{\mathrm{a}}$ & $55,45 \pm 18,58$ & $42,43 \pm 17,7$ \\
\hline ECGl entrada $(\text { média } \pm \text { DP) })^{b}$ & $8,67 \pm 3,23$ & $12,0 \pm 2,88$ \\
\hline ECGl PO $\left(\right.$ média \pm DP) ${ }^{\mathrm{c}}$ & $5,42 \pm 3,12$ & $12,08 \pm 2,86$ \\
\hline Glicemia entrada (média $\pm \mathrm{DP})^{\mathrm{d}}$ & $210,87 \pm 68,3$ & $131,05 \pm 32,72$ \\
\hline Glicemia PO (média \pm DP) $)^{\mathrm{e}}$ & $220,83 \pm 102,06$ & $116,08 \pm 22,08$ \\
\hline PAM entrada $(\text { média } \pm D P)^{\mathrm{f}}$ & $144,13 \pm 36,06$ & $104,65 \pm 18,79$ \\
\hline PAM PO $(\text { média } \pm \text { DP) })^{\mathrm{g}}$ & $117,93 \pm 22,55$ & $95,0 \pm 12,33$ \\
\hline $\begin{array}{l}\text { Tempo de internação } \\
(\text { média } \pm \mathrm{DP})^{\mathrm{h}}\end{array}$ & $18,63 \pm 20,64$ & $30,15 \pm 21,48$ \\
\hline Hematoma lobar & 34 & 31 \\
\hline Hematoma em gânglios da base & 18 & 4 \\
\hline Hematoma cerebelar & 7 & 5 \\
\hline Hematoma em ponte & 1 & - \\
\hline
\end{tabular}

*GOS: Glasgow Outcome Score; M: masculino; F: feminino; DP: desvio-padrão; ECGl: Escala de Coma de Glasgow; PO: 24 horas pós-operatório.

${ }^{a}$ Diferença significativa: $P=0,001 ;{ }^{b}$ Diferença significativa: $P<0,001$;

Diferença significativa: $\mathrm{P}<0,001$; ${ }^{\mathrm{d}}$ Diferença significativa: $\mathrm{P}<0,001$;

Diferença significativa: $\mathrm{P}<0,001$; ${ }^{\mathrm{f}}$ Diferença significativa: $\mathrm{P}<0,001$;

${ }^{\mathrm{g}}$ Diferença significativa: $\mathrm{P}<0,001,{ }^{\mathrm{h}}$ Diferença significativa: $\mathrm{P}=0,008$.

A distribuição por sexo foi similar em ambos os grupos estratificados pelo GOS, com diferença significativa em relação à idade, tendo os pacientes com prognóstico neurológico favorável média etária inferior à média do segundo grupo $(42,43$ anos versus 55,45 anos, $\mathrm{p}=0,001)$.

As pontuações obtidas na ECGl, tanto na entrada quanto em 24 horas de pós-operatório, apresentaram diferenças significativas, com escores maiores no grupo com prognóstico favorável (médias de 8,67 versus 12 na avaliação inicial, $\mathrm{p}<0,001$; e médias de 5,42 versus 12,08 após 24 horas de pós-operatório, $\mathrm{p}<0,001$ ).

Os níveis glicêmicos foram significativamente diferentes em ambos os grupos, com valores maiores para o grupo com evolução desfavorável (médias de $210,87 \mathrm{mg} / \mathrm{dL}$ versus $131,05 \mathrm{mg} / \mathrm{dL}$ na entrada, $\mathrm{p}<$ 0,001 ; e médias de $220,83 \mathrm{mg} / \mathrm{dL}$ versus $116,08 \mathrm{mg} / \mathrm{dL}$ no pós-operatório, $\mathrm{p}<0,001$ ).

Dos pacientes com valor de hiperglicemia na entrada maior que $190 \mathrm{mg} / \mathrm{dL}, 84,4 \%$ faleceram, enquanto naqueles com valores glicêmicos maiores que $200 \mathrm{mg} / \mathrm{dL}$, $240 \mathrm{mg} / \mathrm{dL}$ e $300 \mathrm{mg} / \mathrm{dL}$, os totais de óbitos foram de, respectivamente, $87 \%$, 93\% e 100\%. Com 24 horas pós-operatórias, as porcentagens de óbitos dos pacientes com glicemias maiores que $180 \mathrm{mg} / \mathrm{dL}, 200 \mathrm{mg} / \mathrm{dL}, 240$ $\mathrm{mg} / \mathrm{dL}$ e $300 \mathrm{mg} / \mathrm{dL}$ foram de, respectivamente, $87 \%$, $90 \%, 92 \%$ e $100 \%$.

Os valores da PAM mostraram diferenças estatisticamente significativas entre ambos os grupos, com valores maiores para o grupo de prognóstico desfavorável (médias de 144,13 mmHg versus 104,65 mmHg na entrada, $\mathrm{p}<0,001$; médias de $117,93 \mathrm{mmHg}$ versus $95 \mathrm{mmHg}$ com 24 horas pós-operatório, $\mathrm{p}<0,001$ ).

Em relação ao tempo de internação, observamos diferença significativa entre os grupos $(p=0,008)$, com média de 18,63 dias para o grupo com prognóstico neurológico desfavorável e de 30,15 dias para o grupo com prognóstico favorável.

Quanto à localização do hematoma, no grupo com prognóstico desfavorável (GOS $\leq 3$ ), verificamos a seguinte distribuição: 34 casos de hematoma lobar, 18 em gânglios da base, 1 cerebelar e 1 pontino. No grupo com prognóstico neurológico favorável (GOS $\geq 4$ ), observamos 31 casos de hematoma lobar, 4 nos gânglios da base e 5 cerebelares. Efetuando-se um teste de qui-quadrado entre os dois grupos, verifica-se maior número de hematomas nos gânglios da base no grupo de pior prognóstico.

\section{Discussão}

A elevada taxa de morbidade e de mortalidade associada ao HIPE é causada primariamente pelo efeito mecânico do hematoma, levando a aumento da pressão intracraniana (HIC) e conseqüente diminuição da pressão de perfusão cerebral (PPC) e do fluxo sanguíneo cerebral (FSC), assim como compressão e destruição do tecido nervoso adjacente. Além disso, há complexa desregulação hormonal, com liberação de diversos fatores (como catecolaminas, cortisol e interleucinas), levando a importante desbalanço glicêmico e pressórico ${ }^{19,33}$.

A hiperglicemia como conseqüência ao dano cerebral foi descrita inicialmente em 1849, por Claude Bernard $^{5}$. Tal hiperglicemia representa uma resposta ao evento estressante, refletindo a extensão do dano cerebral $^{12,21,29,35,36}$ e podendo ser importante indicador prognóstico (tabela 2) ${ }^{18,23,25,26,30,36}$.

Os primeiros hormônios glicorreguladores associados a resposta hiperglicêmica são: catecolaminas, glucagon, corticosteróides e somatotropina ${ }^{6,11,16,23}$. As catecolaminas elevam os níveis glicêmicos direta e indiretamente, aumentando a secreção de glucagon e inibindo a secreção de insulina. Além disso, a liberação de interleucina-1 e interleucina- 6 em associação na fase aguda do choque pode ocorrer ${ }^{30}$.

O metabolismo aeróbico converte glicose em piruvato, que entra no ciclo de Krebs como fonte de energia. Supõe-se que, com um aporte excessivo de glicose, em vigência de HIPE, haveria anaerobiose, levando a acúmulo excessivo de lactato, íons hidrogênio e subseqüente acidose tecidual ${ }^{20,34}$.

A acidose intracelular desencadeia uma cascata de eventos, propiciando a entrada de cálcio no espaço intracelular, a liberação de glutamato e ácidos graxos 


\begin{tabular}{|c|c|c|c|}
\hline $\operatorname{Relaça\tilde {a}\square }$ & & abela 2. & \\
\hline Estudo & № de casos & Nível glicêmico & Prognóstico \\
\hline Pentelenyi e Kammerer ${ }^{26}, 1977$ & 331 & $\begin{array}{l}\text { Glicemia }>180 \mathrm{mg} / \mathrm{dL} \\
\text { Glicemia }>240 \mathrm{mg} / \mathrm{dL}\end{array}$ & $\begin{array}{l}\text { Mortalidade }>80 \% \\
\text { Mortalidade de } 100 \%\end{array}$ \\
\hline Pentelenyi et al. ${ }^{25}, 1979$ & 92 & Glicemia $>240 \mathrm{mg} / \mathrm{dL}$ & Mortalidade de $100 \%$ \\
\hline Merguerian et al. $.^{23}, 1981$ & 86 & Glicemia $>270 \mathrm{mg} / \mathrm{dL}$ & Mortalidade de $100 \%$ \\
\hline Young et al. ${ }^{36}, 1989$ & 59 & Glicemia $>200 \mathrm{mg} / \mathrm{dL}$ & Diferença significativa \\
\hline Lam et al. ${ }^{18}, 1991$ & 169 & Glicemia $>200 \mathrm{mg} / \mathrm{dL}$ & Valor preditivo com pior prognóstico \\
\hline Vasconcellos et al., 2006 & 100 & Glicemia $>300 \mathrm{mg} / \mathrm{dL}$ & Mortalidade de $100 \%$ \\
\hline
\end{tabular}

e, por fim, a destruição de células neuronais. O excesso de íons hidrogênio pode aumentar a produção de radicais livres ${ }^{28,32}$.

Nossos resultados mostram que o grupo de pacientes com prognóstico neurológico desfavorável apresentou níveis glicêmicos significativamente mais altos do que os apresentados no grupo com prognóstico favorável, sugerindo que a hiperglicemia tenha relação direta com a injúria neurológica.

Os efeitos secundários da hiperglicemia sabidamente pioram a lesão neuronal. Assim, orientamos que seja feito controle estrito da glicemia com insulinoterapia nos primeiros dias do HIPE. É de extrema importância manter a glicemia entre 80 e $120 \mathrm{mg} / \mathrm{dL}$, realizando monitorização nas primeiras 48 a 72 horas em todos os pacientes em intervalos de 6 horas, evitando-se soluções parenterais de glicose. O suporte nutricional deve ser iniciado no menor prazo possível e, de preferência, por via oral ou enteral. Devem-se evitar e combater causas adicionais de hiperglicemia, como o uso de esteróides, processos infecciosos, além de outros distúrbios metabólicos ou mesmo a hipoglicemia.

O FSC é determinado fisiologicamente pela PPC, $\mathrm{PaCO}_{2}, \mathrm{PaO}_{2}$ e pela atividade metabólica cerebral. A PPC é definida como a diferença entre a PAM e a pressão intracraniana (PIC). Tais valores estão relacionados em um sistema de auto-regulação que mantém constante o FSC dentro de um padrão de normalidade.

Após um evento de HIPE, há rápido aumento da PIC, e a maioria dos pacientes torna-se hipertensa, mesmo aqueles sem antecedente mórbido de HA. Além desse fator, estresse da doença aguda, dor, náuseas, vômitos, repleção vesical, HA prévia ou resposta cerebral ao choque favorecem a manutenção de HA. As recomendações para o tratamento da fase aguda do HIPE, segundo preconizado pelo Acute Stroke Collaboration e American Heart Association ${ }^{4}$, estão sumarizados na tabela 3 .

\begin{tabular}{lll}
\hline \multicolumn{3}{c}{ Controle da pressão arterial na fase aguda do HIPE ${ }^{14}$} \\
\hline Pressão arterial & $\begin{array}{l}\text { HIPE com doença } \\
\text { associada }\end{array}$ & Apenas HIPE \\
\hline PAD $>140 \mathrm{mmHg}$ & Opção 1 & Opção 1 ou 2 \\
PAS $>220 \mathrm{mmHg}$ ou & Opção 1 ou 2 & Opção 2 \\
PAM $>130 \mathrm{mmHg}^{\mathbf{a}}$ & & \\
PAS $>160 \mathrm{mmHg}$ ou $^{\text {PAD }}>105 \mathrm{mmHg}^{\mathrm{a}}$ & Opção 2 & Opção 2 \\
\hline
\end{tabular}

PAD: pressão arterial diastólica; PAS: pressão arterial sistólica.

aDuas medidas com intervalo de 10 minutos. Reduzir

PAM, no máximo, em $30 \%$ do inicial.

${ }^{b}$ Doença associada (encefalopatia hipertensiva, angina,

dissecção aórtica, insuficiência cardíaca).

Opção 1: nitroprussiato de sódio $(1 \mathrm{amp}=50 \mathrm{mg})$. A droga poderá

ser diluída em solução de glicose a $5 \%$. Usar 0,5 a 8 ug $/ \mathrm{kg} / \mathrm{min}$,

fazendo reajustes, se necessário, a cada 10 minutos.

Opção 2A: metoprolol ( $1 \mathrm{amp}=5 \mathrm{~mL}, 1 \mathrm{mg} / \mathrm{mL})$. Aplicar EV: $5 \mathrm{mg}$

a cada $10 \mathrm{minutos}$, sendo $1 \mathrm{mg} / \mathrm{min}$. Máximo de $20 \mathrm{mg}$.

Opçãp 2B: enalapril $(1 \mathrm{amp}=1 \mathrm{~mL}=1 \mathrm{mg} / \mathrm{mL})$. Correr $1 \mathrm{mg}$ em, no mínimo, 5

minutos a 1 hora. Caso necessário, essa dose pode ser repetida após 1 hora. Novas

doses, porém, devem ser dadas num intervalo mínimo de 6 horas. Manutenção: 5

a $40 \mathrm{mg} / \mathrm{dia}$, dose única ou em duas tomadas (comprimidos de 5, 10 e $20 \mathrm{mg}$ ).

Como demonstrado nos resultados deste estudo, o grupo de pacientes com prognóstico desfavorável apresentou valores significativamente maiores de PAM do que o grupo com prognóstico favorável, sugerindo o efeito prejudicial da hipertensão no prognóstico neurológico de pacientes com HIPE.

A rápida normalização da PAM pode ser extremamente danosa ao paciente, pois pode desencadear redução da PPC com surgimento de áreas de isquemia. Deste modo, o tratamento da hipertensão arterial deve ser cauteloso, mas iniciado o mais rapidamente possível, procurando manter a PAM entre 125 e $135 \mathrm{mmHg}$.

Observamos diferença significativamente maior, com relação à idade, no grupo de pior prognóstico neurológico, sugerindo que aqueles com idade principalmente acima de 55 anos apresentam mais complicações e pior resultado cirúrgico. A literatura refere que a faixa etária mais acometida é aquela entre 30 e 80 anos, com predomínio na sétima e quinta décadas ${ }^{31}$. Nosso estudo mostrou que os pacientes mais jovens, principalmente aqueles com idade 
inferior a 40 anos, e com escore de admissão superior a 11 na ECGl obtiveram evolução satisfatória. Trabalhos da literatura apontam alta mortalidade (próxima a 100\%) nos pacientes admitidos com escore inferior a 6 , independentemente do tratamento adotado ${ }^{3,9,21,23,25,26}$. Assim, o grupo de pacientes com pior prognóstico teve, em relação ao tempo de internação, diferença significativamente menor, ou seja, faleceram precocemente, reduzindo sua média de permanência hospitalar em relação ao grupo com melhor prognóstico neurológico.

Quanto à localização, os hematomas foram mais freqüentes em regiões superficiais (lobar), cortical ou subcortical, acometendo $65 \%$ dos pacientes $(56,6 \%$ no grupo de pior prognóstico e $77,5 \%$ no grupo com melhor prognóstico neurológico). Trabalhos da literatura apontam os hematomas profundos como sendo os mais comuns (freqüência de $60 \%$ e de apenas $30 \%$ para os lobares) ${ }^{3,7,30}$. A diferença dos achados neste estudo comparados com dados da literatura deve-se ao fato de que nestes últimos se incluem casos cirúrgicos e não cirúrgicos, enquanto em nosso estudo foram considerados somente os casos cirúrgicos, o que mostra relação maior de hematomas lobares, uma vez que os hematomas profundos geralmente não têm indicação cirúrgica. Ou ainda, nessas situações, a cirurgia é indicada quando se trata de hematomas volumosos, o que talvez justifique o maior número de casos dessa localização no grupo de pior prognóstico.

Com relação ao tratamento, ainda existe muita controvérsia a respeito do tipo mais adequado para cada paciente ${ }^{2}$, se clínico ou cirúrgico, e qual o tipo de abordagem cirúrgica. Muitas vezes, a drenagem cirúrgica do hematoma é insatisfatória. Esse fato ocorre porque, conforme visto em estudos experimentais, o tecido cerebral que circunda o hematoma desenvolve isquemia $\mathrm{e}$ edema que pode exercer o efeito do volume do próprio hematoma, causando danos irreversíveis. As indicações para o tratamento cirúrgico são: hemorragias superficiais, com volume entre $20 \mathrm{~mL}$ e $80 \mathrm{~mL}$, piora neurológica em curto espaço de tempo, pacientes jovens com poucas comorbidades, desvio de estruturas da linha média, hematomas cerebelares $\geq$ a $3 \mathrm{~cm}$ no maior diâmetro e presença de hidrocefalia à montante, além das contraindicações relativas em relação à presença de hematomas pequenos em pacientes alertas ou moribundos ${ }^{15}$.

\section{Conclusão}

A hiperglicemia e a hipertensão arterial são componentes freqüentes das lesões neurológicas, sendo indicadores significativos de gravidade e confiáveis na avaliação do prognóstico neurológico nos pacientes com hematoma intraparenquimatoso espontâneo.

\section{Referências}

1. AGUIAR PH, MANDEL M, LOURENCCO L, FONTES R, MELGARIS M, SIMM R et al.: Hemorragia intracerebral espontânea. O que há de novo. J Bras Neurocirurg 12:126-38, 2001.

2. AUER LM, DEINSBERGER W, NIEDERKORN K, GELL G, KLEINERT K, SCHNEIDER $G$ et al.: Endoscopic surgery versus medical treatment for spontaneous intracerebral hematoma: a randomized study. J Neurosurg 70:530-5, 1989.

3. AYAD PB, AWAD IA: Surgery for intracerebral hemorrhage. Neurology 51:69-73, 1998.

4. BATH FJ, BATH PMW: What is the correct management of blood pressure in acute stroke? The blood pressure in Acute Stroke Collaboration. Cerebrovasc Dis 7:205-13, 1997.

5. BERNARD C: Chiens rendus diabétiques. C R Soc Biol (Paris) I:60, 1849.

6. BESSEY PQ, WATTERS JM, AOKI TT, WILMORE DW: Combined hormonal infusion simulates the metabolic response to injury. Ann Surg 200:264-81, 1984.

7. BOONY AS, DENNIS M, SANDERCOCK P, BAMFORD J, BURN J, WARLOW C: Primary intracerebral hemorrhage in the Oxfordshire Community Stroke Project: Incidence, clinical features and cause. Cerebrovasc Dis 3:343-9, 1993.

8. BRODERICK JP, BROTTT, TOMSICK T, MILLER R, HUSTER $\mathrm{G}$ : Intracerebral hemorrhage more than twice as common as subarachnoid hemorrhage. J Neurosurg 78:188-91, 1993.

9. COLOMBO A, CHELDI A, AMICO G: 52 cases of spontaneous cerebral hemorrhage. Riv Neurol 56:383-95, 1986.

10. CUNNINGHAM AS, SALVADOR R, COLES JP, CHATFIELD DA, BRADLEY PG, JOHNSTON AJ et al:: Physiological thresholds for irreversible tissue damage in contusional regions following traumatic brain injury. Brain 128:1931-4, 2005.

11. De COURTEN-MYERS GM, KLEINHOLZ M, HOLM P, De VOE G, SCHMITT G, WAGNER KR et al.: Hemorrhagic infarct conversion in experimental stroke. Ann Emerg Med 21:120-6, 1992.

12. De SALLES AAF, MUIZELAAR JP, YOUNG HF: Hyperglycemia, cerebrospinal fluid lactic acidosis, and cerebral blood flow in severely head-injured patients. Neurosurgery 21:45-50, 1987.

13. FERNANDES HN, GREGSON B, SIDDINIQUE G, MENDELOW AD: Surgery in intracerebral hemorrhage, the uncertainty continues. Stroke 31:2511-6, 2000.

14. GAGLIARDI RJ, RAFFIN CN, CABETTE SRF: Primeiro Consenso Brasileiro do Tratamento da Fase Aguda do Acidente Vascular Cerebral. Arq Neuropsiquiatr (São Paulo) 59:972-80, 2001.

15. GUIMARÃES OM, TOLEDO DER, VENTURELLI ID, NEVES MV, GUIMARÃES MD: Hematomas encefálicos não traumáticos - critérios de tratamento. J Bras Neurocirurg 13:49-56, 2002

16. HOWARD JM: Studies of absorption and metabolism of glucose following injury. Ann Surg 141:321-6, 1955.

17. JENETT B, BOND M: Assessment of outcome after severe brain damage: a practical scale. Lancet 1:480-4, 1975.

18. LAM AM, WINN HR, CULLEN BF, SUNDLING N: Hyperglycemia and neurological outcome in patients with head injury. J Neurosurg 75:545-5, 1991.

19. LEVINE SR, WELCH KMA: Cocaine and stroke. Stroke 19:779-83, 1988.

20. MARSH WR, ANDERSON RE, SUNDT TM Jr. Effect of hyperglycemia on brain $\mathrm{pH}$ levels in areas of focal incomplete cerebral ischemia in monkeys. J Neurosurg 65:693-6, 1986.

21. MASE G, ZORZON M, BIASUTTI E, TASCA G, VITRANI 
B, CAZZATO G: Immediate prognosis of primary intracerebral hemorrhage using an easy model for the prediction of survival. Acta Neurol Scand 91:306-9, 1995.

22. MCCLAIN C, COHEN D, PHILLIPS R: Increased plasma and ventricular fluid interleukin- 6 levels in patients with head injury. J Lab Clin Med 118:225-31, 1991.

23. MERGUERIAN PA, PEREL A, WALD U, FEISOND M, COTEV S: Persistent nonketotic hyperglycemia as a grave prognostic sign in head-injured patients. Crit Care Med 9:838-40, 1981

24. MOHR JP, CAPLAN LR, MELSKI JW et al.: The Harward Cooperative Stroke Registry: A prospective registry. Neurology 28:754-62, 1978.

25. PENTELENYI T, KAMMERER L, STUTZEL M, BALAZSI I: Alterations of the basal serum insulin and blood glucose in brain-injured patients. Injury 10:201-8, 1979.

26. PENTELENYIT, KAMMERER L: Changes in blood glucose after head injury and its prognostic significance. Injury 8:264-8, 1977.

27. POUNGVARIN N, VIRIYAVEJAKUL A: Spontaneous supratentorial intracerebral hemorrhage: a prognostic study. J Med Assoc Thai 73:206-11, 1990.

28. REHNCRONA S, HAUGE HN, SIESJÖ BK: Enhancement of iron-catalyzed free radical formation by acidosis in brain homogenates: Differences in effect by lactic acid and $\mathrm{CO}_{2}$. J Cereb Blood Flow Metab 9:65-70, 1989.

29. ROSNER MJ, NEWSOME HH, BECKER DP: Mechanical brain injury: The sympathoadrenal response. J Neurosurg 61:76-86, 1984.

30. ROVLIASA, KOTSOU S: The influence of hyperglicemia on neurological outcome in patients with severe head injury. Neurosurgery 46:335-43, 2000.
31. SACCO RL, MAYER AS: Epidemiology of intracerebral hemorrhage. In Feldman E (ed): Intracerebral Hemorrhage. Armonk, Futura, 1994, pp 3-23.

32. SIESJÖ BK, WIELOCH T: Cerebral metabolism in ischemia: Neurochemical basis for therapy. $\mathrm{Br} J$ Anaesth 57:47-62, 1985.

33. SLOAN MA, KITTNER SJ, RIGAMONTI D, PRICE TR: Occurrence of stroke associated with use/abuse of drugs. Neurology 41:1358-64, 1991.

34. SMITH ML, von HANWEHR R, SIESJÖ BK: Changes in extra- and intracellular $\mathrm{pH}$ in the brain during and following ischemia in hyperglycemic and moderately hypoglycemic rats. J Cereb Blood Flow Metab 6:574-83, 1986.

35. SPITZER K, THIE A, KUNZE K: Incidence and prognosis of internal medicine complications in spontaneous intracranial hematomas. Nervenarzt 59:647-53, 1988.

36. YOUNG B, OTT L, DEMPSEY R, HAACK D, TIBBS P: Relationship between admission hyperglycemia and neurologic outcome of severely brain-injured patients. Ann Surg 210:466-73, 1989.

Original recebido em março de 2006

Aceito para publicação em maio de 2006

\section{Endereço para correspondência}

Lucas Perez de Vasconcellos

Rua Desembargador Joaquim Barbosa de Almeida, 368

05463-010 - São Paulo, SP

E-mail:lucasvasconcellos@hotmail.com 